\title{
ON FINITE DEFORMATIONS OF SPATIALLY CURVED BISYMMETRIC THIN-WALLED RODS
}

\author{
R. BIJAK ${ }^{1}$, G. KOLODZIEJ ${ }^{2}$
}

\begin{abstract}
Deriving the formulas for strain components, we are assuming, that cross-section of a rod being rotated in space during deformation does not need to be perpendicular to deformed centroid line. This not a quite intuitive assumption allows for more compact and easier formulas for strain tensor or equilibrium equations. Derived transformations between actual and initial coordinate system, components of strain tensor and virtual works principle for investigated spatially curved beams of bisymmetric cross-section are shown in this paper. Conformity with other models from referenced literature is also shown.
\end{abstract}

Keywords: space-curved thin-walled rods, bisymmetric cross-sections, finite deformations, second-order approximations of finite rotations, Reissner model, Bernoulli hypothesis

\section{INTRODUCTION}

Most of the finite element formulations are concerned with rods where centre of gravity and shear centre coincide, which happens most frequently in practice. The problem of coupled bending torsion deformation of beams has been studied theoretically, e.g. Reissner [2,3]. Simo, Vu-Quoc [8] incorporated torsion-warping deformation and the associated finite element formulation with finite rotations. Gruttmann et al. [9] developed elastoplastic analysis of the finite element formulation of a three dimensional eccentric space curved beams with arbitrary cross-section including warping, where the strain measures were derived by exploitation of the Green-Lagrange strain tensor. Battini and Pacoste [10] presented the formulation of 3D co-rotational beam elements for the non-linear

\footnotetext{
${ }^{1}$ DSc., Kielce University of Technology, Faculty of Civil Engineering and Architecture, Al. 1000-lecia PP 7, 25-314 Kielce, Poland, e-mail: r.bijak@wp.pl

${ }^{2}$ MSc., Kyotec Group, Batalionu Platerówek 3, 03-308 Warsaw. Poland, e-mail: grzegorz.kolodziej@kyotecgrup.com
} 
and stability analysis of frame structures, pointing differences related to warping effects and parameterization of finite 3D rotations and the other is related to warping effects. Saleeb et al. [13] and $\mathrm{Kim}$ et al. $[12,13]$ showed effective formulation of spatial buckling of thin-walled frames employing second-order approximation of finite rotations. Reissner presented [6] computational model of spatially curved solid rod valid in range of small rotations of the rod's section. Improved displacement field of curved beams, obtained by considering the second-order terms of finite rotations was presented by Saleeb and Gendy [13], Hu et al. [14]. Pi, Trahair presented flexuraltorsional buckling of circular arches [17] and non-linear behavior of elastic circular arches [19]. In case of spatially curved beams things are much more complicated comparing to planar curvature cases [15].

For open-section beams made from isotropic material we assume Bernoulli hypothesis, shear stresses coming from bending and warping are ignored, only those due to St.Venant torsion are considered. Formulas for components of strain tensor and equilibrium for beams curved in space shown in Abaqus theory manual [5] were employed. This general formulation originating in deformation gradient of spatially curved, thin-walled beams allows for derivation of practically any engineering theory of thin-walled beams presented in quoted references.

There are, however, two essential differences between theory shown in Abaqus [5] and other engineering theories:

- instead of finite rotations we are dealing with second order approximation of finite rotations

- in many of these theories it is assumed Bernoulli hypothesis and shear stresses coming from section warping are neglected

\section{KINEMATICS AND STRAINS OF THIN-WALLED ROD}

A beam with reference configuration according to Figure 1 is considered. Although in general case centroid $\mathrm{G}$ and the centre of shear $\mathrm{S}$ are independent, for bisymmetric cross-section considered in the presented work they coincide. The first considered system is curved and twisted spatial material reference system $\mathbf{T}_{i}$ tied to cross section of rod before deformation. The system is built by assuming first, that unit vector $\mathbf{T}_{1}$ is aligned to tangent to reference centroid curve of the rod's sections (further the reference curve is identified with the axis of the rod, S is arc-length coordinate along the curve, before deformation). Two mutually perpendicular base vectors $\mathbf{T}_{2}, \mathbf{T}_{3}$ then lie in plane perpendicular to the reference curve and coincide with two principal axes of the cross-section. 
The second local (actual) system is moving system $\mathbf{t}_{i}$ tied to the deformed section. Note, that in general, the vector $\mathbf{t}_{1}$ does not need to be tangent vector of the deformed reference curve.

Transition between the initial and actual basis due to rotation of deformed section can be written using orthogonal transformation:

$$
\mathbf{t}_{i}(S)=\mathbf{C}(S) \mathbf{T}_{i}(S)
$$

Position vector of any material point $M\left(S, X_{2}, X_{3}\right)$ in cross-section of thin-walled rod in undeformed configuration may be shown as:

$$
\mathbf{R}_{M}\left(S, X_{2}, X_{3}\right)=\mathbf{R}(S)+X_{2} \mathbf{T}_{2}(S)+X_{3} \mathbf{T}_{3}(S)
$$

Displacement due to warping of the twisted section is defined in direction of versor $\mathbf{t}_{1}$ as product of unknown warping amplitude $p(S)$ and given warping function $\omega\left(X_{2}, X_{3}\right)$. Consequently, position vector of any material point $M\left(S, X_{2}, X_{3}\right)$ in cross-section of thin walled rod after deformation is given as:

$$
\mathbf{r}_{M}\left(S, X_{2}, X_{3}\right)=\mathbf{r}(S)+X_{2} \mathbf{t}_{2}(S)+X_{3} \mathbf{t}_{3}(S)-\omega\left(X_{2}, X_{3}\right) p(S) \mathbf{t}_{1}(S)
$$

Position vector of points on reference curve is designated as $\mathbf{R}(S)$ for initial configuration and $\mathbf{r}(S)$ for actual configuration.
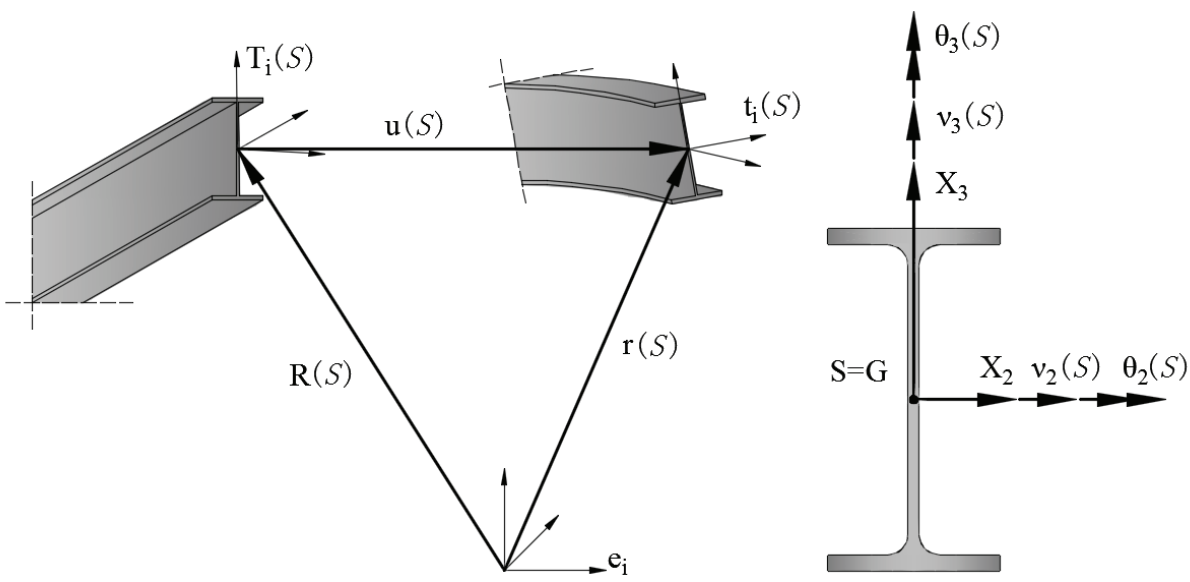

Fig. 1. Initial and actual configuration of the rod. 
Equations $(2.4 \div 6)$ show formulas which are used to define the components of strain tensor. In initial, undeformed configuration with basis $\left[\mathbf{T}_{1}, \mathbf{T}_{2}, \mathbf{T}_{3}\right]$, we have:

$$
B(S)=\mathbf{T}_{3} \cdot \mathbf{T}_{2}^{(1)}, \quad K_{2}(S)=\mathbf{T}_{1} \cdot \mathbf{T}_{3}^{(1)}, \quad K_{3}(S)=-\mathbf{T}_{1} \cdot \mathbf{T}_{2}^{(1)}
$$

where: $f^{(\mathrm{k})}=\partial^{\mathrm{k}} f / \partial S^{\mathrm{k}}-$ denotes $k$-th derivative of function with respect to arc-length coordinate $S$ measured on reference curve before deformation, $\mathbf{a} \cdot \mathbf{b}-$ is scalar product of vectors $\mathbf{a}$ and $\mathbf{b}$.

Similarly, in deformed configuration with basis $\left[\mathbf{t}_{1}, \mathbf{t}_{2}, \mathbf{t}_{3}\right]$ :

$$
\begin{aligned}
& b(S)=\mathbf{t}_{3} \cdot \mathbf{t}_{2}^{(1)}, \quad k_{2}(S)=\mathbf{t}_{1} \cdot \mathbf{t}_{3}^{(1)}, \quad k_{3}(S)=-\mathbf{t}_{1} \cdot \mathbf{t}_{2}^{(1)} \\
& \gamma_{1}(S)=\mathbf{t}_{1} \cdot \mathbf{r}^{(1)}-1, \quad \gamma_{2}(S)=\mathbf{t}_{2} \cdot \mathbf{r}^{(1)}, \quad \gamma_{3}(S)=\mathbf{t}_{3} \cdot \mathbf{r}^{(1)}
\end{aligned}
$$

Reissner [6] uses the formula (2.6a) directly, while in ABAQUS theory [5] approximation: $\mathbf{t}_{1} \cong \mathbf{s}$ is used, where $\mathbf{s}$ is tangent vector to reference curve in deformed configuration given as:

$$
\mathbf{s}=\lambda^{-1} \mathbf{r}^{(1)}, \quad \lambda=\left\|\mathbf{r}^{(1)}\right\|
$$

where: $\lambda$ denotes the axial stretch and \|\| length of vector.

Considering the above, we obtain:

$$
\gamma_{1}(S)=\lambda-1
$$

Please note, however, Abaqus theory makes use of the following assumption:

$$
\gamma_{1}(S)=\ln \lambda
$$

but for $\lambda \approx 1$ we can assume with enough accuracy $\ln \lambda \approx \lambda-1$.

Axial strain is given as:

$$
\varepsilon_{11}=\gamma_{1}-X_{2}\left(k_{3}-K_{3}\right)+X_{3}\left(k_{2}-K_{2}\right)-\omega p^{(1)}+\frac{1}{2}\left(X_{2}^{2}+X_{3}^{2}\right)\left(b^{2}-B^{2}\right)
$$

In case the terms coming from Wagner's effect are averaged for the whole section, axial strains can be expressed with following formulas (2.10): 


$$
\begin{aligned}
& \varepsilon_{11}=e-X_{2}\left(k_{3}-K_{3}\right)+X_{3}\left(k_{2}-K_{2}\right)-\omega p^{(1)} \\
& e=\gamma_{1}+\frac{1}{2} \frac{I_{p}}{A}\left(b^{2}-B^{2}\right), \quad I_{p}=I_{22}+I_{33}
\end{aligned}
$$

where (Fig.1):

$A$ - cross-section area,

$I_{22}$ - principal moment of inertia about axis 2 ,

$I_{33}$ - principal moment of inertia about axis 3 .

Shear strains are split as follows:

$$
\gamma_{12}=\gamma_{2}+\gamma_{12}^{T}, \quad \gamma_{13}=\gamma_{3}+\gamma_{13}^{T}
$$

where: $\gamma_{2}$ and $\gamma_{3}-$ according to (2.6bc).

Shear strains coming from twist of the section are given as:

(2.12ab) $\quad \gamma_{12}^{T}=-\left(X_{3}+\omega_{, 2}\right)(b-B)+\omega_{, 2} p_{w}, \quad \gamma_{13}^{T}=\left(X_{2}-\omega_{, 3}\right)(b-B)+\omega_{, 3} p_{w}$

Similarly, for total warping, there is distinction made for free $(b-B)$ and constrained warping $\left(p_{w}\right)$ :

$$
p_{w}=(b-B)-p
$$

In Abaqus [5] the equation (1) is considered with respect to quaternions algebra, while common engineering approach is to use approximations of some kind:

$$
\mathbf{t}_{i}=C_{k i} \mathbf{T}_{k}
$$

In above equation (2.14) Einstein summation notation is used.

Particularly, one can find the formula tor transformation $\left[C_{\mathrm{ij}}\right]$ given in [16] by Van Erp et. al:

$$
(2.15)\left[C_{i j}\right]=\left[\begin{array}{ccc}
1-\frac{1}{2}\left(\theta_{2}^{2}+\theta_{3}^{2}\right) & -\theta_{3} \cos \varphi+\theta_{2} \sin \varphi & \theta_{3} \sin \varphi+\theta_{2} \cos \varphi \\
\theta_{3} & \left(1-\frac{1}{2} \theta_{3}^{2}\right) \cos \varphi+\frac{1}{2} \theta_{2} \theta_{3} \sin \varphi & -\left(1-\frac{1}{2} \theta_{2}^{2}\right) \sin \varphi+\frac{1}{2} \theta_{2} \theta_{3} \cos \varphi \\
-\theta_{2} & \left(1-\frac{1}{2} \theta_{2}^{2}\right) \sin \varphi+\frac{1}{2} \theta_{2} \theta_{3} \cos \varphi & \left(1-\frac{1}{2} \theta_{2}^{2}\right) \cos \varphi-\frac{1}{2} \theta_{2} \theta_{3} \sin \varphi
\end{array}\right]
$$


where: $\varphi(S), \theta_{2}(S), \theta_{3}(S)$ are functions of arc-length coordinate $S$, which can be interpreted as angles of rotation about axes 1,2,3 (fig. 1) only in case of linear approximation of transformation matrix $\mathbf{C}$.

\section{INTERNAL VIRTUAL WORK AND DEFINITION OF STRESS RESULTANTS}

Generalized internal forces, in case of isotropic material may be given as:

$$
\begin{gathered}
N=\int_{A} \sigma_{11} d A=E A e \\
F_{2}=\int_{A} \tau_{12} d A=G A_{v 3} \gamma_{2} \\
F_{3}=\int_{A} \tau_{13} d A=G A_{v 2} \gamma_{3} \\
M_{2}=\int_{A} X_{3} \sigma_{11} d A=E I_{22}\left(k_{2}-K_{2}\right) \\
M_{3}=-\int_{A} X_{2} \sigma_{11} d A=E I_{33}\left(k_{3}-K_{3}\right) \\
B_{w}=\int_{A} \omega \sigma_{11} d A=-E I_{w} p^{(1)}
\end{gathered}
$$

In the above equations: $E, G$ - Young's and shear modulus, $I_{w}$ - warping constant.

The total torque moment $T$, relative to the centroid of the section:

$$
T=\int_{A}\left(\tau_{13} X_{2}-\tau_{12} X_{3}\right) d A=M_{t}+M_{p}
$$

is further split into two parts: St. Venant torsional moment $M_{t}$ and warping moment $M_{p}$ :

$$
\begin{gathered}
M_{t}=\int_{A}\left[\tau_{13}\left(X_{2}-\omega_{, 3}\right)-\tau_{12}\left(X_{3}+\omega_{, 2}\right)\right] d A=G I_{T}(b-B) \\
M_{p}=\int_{A}\left(\tau_{13} \omega_{, 3}+\tau_{12} \omega_{, 2}\right) d A=G\left(I_{p}-I_{T}\right) p_{w}
\end{gathered}
$$

St. Venant torsion constant $I_{T}$ is calculated in the following way:

$$
I_{T}=\int_{A}\left[\left(X_{2}-\omega_{, 3}\right)^{2}+\left(X_{3}+\omega_{, 2}\right)^{2}\right] d A
$$


Virtual work principle for internal forces may be written as:

$$
\delta \Pi=\int_{V}\left(\sigma_{11} \delta e_{11}+\tau_{12} \delta \gamma_{12}+\tau_{13} \delta \gamma_{13}\right) d V
$$

Considering all above we have the following formula:

$$
\delta \Pi=\int_{L}\left(N \delta e+F_{2} \delta \gamma_{2}+F_{3} \delta \gamma_{3}+M_{2} \delta k_{2}+M_{3} \delta k_{3}+M_{t} \delta b+M_{p} \delta p_{w}-B_{w} \delta p^{(1)}\right) d x
$$

\section{EXTENSIONS OF REISSNER'S MODEL [6]}

The original approach of Reissner's model [6] is to employ the differential equations of equilibrium of internal forces and other parameters of basis vector transformations as well. Basis of extension of mentioned Reissner's formulation is to take into consideration also the warping of the section. The basic approximation (2.15) is to use simple estimation of trigonometric functions:

$$
\sin \varphi \approx \varphi, \quad \cos \varphi \approx 1-\varphi^{2} / 2
$$

It leads to following transformation:

$$
\left[C_{i j}\right]=\left[\begin{array}{ccc}
1-\left(\theta_{2}^{2}+\theta_{3}^{2}\right) / 2 & -\theta_{3}+\theta_{2} \varphi & \theta_{2}+\theta_{3} \varphi \\
\theta_{3} & 1-\left(\theta_{3}^{2}+\varphi^{2}\right) / 2 & -\varphi+\theta_{2} \theta_{3} / 2 \\
-\theta_{2} & \varphi+\theta_{2} \theta_{3} / 2 & 1-\left(\theta_{2}^{2}+\varphi^{2}\right) / 2
\end{array}\right]
$$

Substituting $(2.14,4.2)$ into $(2.5)$ one obtains:

$$
\begin{gathered}
b=\varphi^{(1)}+\left(\theta_{2}^{(1)} \theta_{3}-\theta_{2} \theta_{3}^{(1)}\right) / 2+\left(1-\theta_{2}^{2} / 2-\theta_{3}^{2} / 2\right) B+\theta_{3} K_{2}-\theta_{2} K_{3} \\
k_{2}=\theta_{2}^{(1)}+\theta_{3}^{(1)} \varphi+\left(-\theta_{3}+\theta_{2} \varphi\right) B+\left(1-\theta_{3}^{2} / 2-\varphi^{2} / 2\right) K_{2}+\left(\varphi+\theta_{2} \theta_{3} / 2\right) K_{3} \\
k_{3}=\theta_{3}^{(1)}-\theta_{2}^{(1)} \varphi+\left(\theta_{2}+\theta_{3} \varphi\right) B+\left(-\varphi+\theta_{2} \theta_{3} / 2\right) K_{2}+\left(1-\theta_{2}^{2} / 2-\varphi^{2} / 2\right) K_{3}
\end{gathered}
$$

These are equal to expressions (21abc) of [6] (with denotation: $\varphi=\varphi_{t}, \theta_{2}=-\varphi_{2}, \theta_{3}=\varphi_{1}, B=1 / \rho_{t}, K_{2}=-$ $\left.1 / \rho_{2}, K_{3}=1 / \rho_{1}\right)$.

We will show the updated position vector of reference curve $\mathbf{r}(\mathbf{s})$ during deformation expressed in terms of displacements in basis $\mathbf{T}_{i}$ :

$$
\mathbf{r}(S)=\mathbf{R}(S)+u(S) \mathbf{T}_{1}(S)+v_{2}(S) \mathbf{T}_{2}(S)+v_{3}(S) \mathbf{T}_{3}(S)
$$


On basis of (4.4) the following can be written:

$$
\mathbf{r}^{(1)}=\left(1+\widehat{u}^{(1)}\right) \mathbf{T}_{1}+\widehat{v}_{2}^{(1)} \mathbf{T}_{2}+\widehat{v}_{3}^{(1)} \mathbf{T}_{3}
$$

(4.6abc)

$$
\widehat{u}^{(1)}=u^{(1)}-v_{2} K_{3}+v_{3} K_{2}, \quad \widehat{v}_{2}^{(1)}=v_{2}^{(1)}+u K_{3}-v_{3} B, \quad \widehat{v}_{3}^{(1)}=v_{3}^{(1)}-u K_{2}+v_{2} B
$$

Substituting (4.5) and (4.6) into (2.6bc):

$$
\begin{aligned}
& \gamma_{2}=-\theta_{3}+\widehat{v}_{2}^{(1)}+\theta_{2} \varphi-\theta_{3} \widehat{u}^{(1)}+\varphi \widehat{v}_{3}^{(1)} \\
& \gamma_{3}=\theta_{2}+\widehat{v}_{3}^{(1)}+\theta_{3} \varphi+\theta_{2} \widehat{u}^{(1)}-\varphi \widehat{v}_{2}^{(1)}
\end{aligned}
$$

Generalized axial strains can be determined using (2.8), and making appropriate substitutions from $(4.5,4.6)$ into $(2.7 b)$ and then into $(2.8)$ :

$$
\gamma_{1}=\sqrt{\left(1+\widehat{u}^{(1)}\right)^{2}+\left(\widehat{v}_{2}^{(1)}\right)^{2}+\left(\widehat{v}_{3}^{(1)}\right)^{2}}-1 \cong \widehat{u}^{(1)}+(1 / 2)\left(\widehat{v}_{2}^{(1)}\right)^{2}+(1 / 2)\left(\widehat{v}_{3}^{(1)}\right)^{2}
$$

\section{PI, TRAHAIR, BRADFORD MODEL $[19,20]$}

On basis of above we can demonstrate that transition to approach shown in Pi, Bradford [20] is possible. In our model we are assuming transformation matrix, which takes into account finite rotation angles (2.15). On such basis we are able to determine generalized strains (2.5). Moreover we are neglecting the shear strains coming from warping $\left(p_{w}=0\right)$, which leads to relationship $p=b$. Assuming $B=0, K_{2}=1 / R, K_{3}=0$ (no initial twist, beam curved in plane) and making substitutions into (4.6) we have:

$(5.1 \mathrm{abc})$

$$
\widehat{u}^{(1)}=u^{(1)}+\frac{v_{3}}{R}, \quad \widehat{v}_{2}^{(1)}=v_{2}^{(1)}, \quad \hat{v}_{3}^{(1)}=v_{3}^{(1)}-\frac{u}{R}
$$

Considering Bernoulli hypothesis: $\mathbf{s}=\mathbf{t}_{1}$. 
The unit vector $\mathbf{s}$, tangent to reference curve after deformation and unit basis vector $\mathbf{t}_{1}$ can be derived as:

$$
\mathbf{s}=\frac{1}{1+\gamma_{1}}\left[\begin{array}{c}
1+\hat{u}^{(1)} \\
\hat{v}_{2}^{(1)} \\
\hat{v}_{3}^{(1)}
\end{array}\right] \cong\left[\begin{array}{c}
1 \\
\hat{v}_{2}^{(1)} \\
\widehat{v}_{3}^{(1)}
\end{array}\right], \quad \mathbf{t}_{1} \cong\left[\begin{array}{c}
1 \\
\theta_{3} \\
-\theta_{2}
\end{array}\right]
$$

Comparing corresponding components in equations (5.2ab) we have:

$$
\theta_{2} \cong-\widehat{v}_{3}^{(1)}, \quad \theta_{3} \cong \widehat{v}_{2}^{(1)}
$$

Substituting (5.3) into $(2.15)$, then into $(2.14,2.5)$ and finally into $(2.10 \mathrm{a})$ it can be written:

$$
\begin{aligned}
\varepsilon_{11}= & u^{(1)}+\frac{v_{3}}{R}+\frac{1}{2}\left(v_{2}^{(1)}\right)^{2}+\frac{1}{2}\left(v_{3}^{(1)}-\frac{u}{R}\right)^{2}-X_{2}\left[v_{2}^{(2)} \cos \varphi+\left(v_{3}^{(2)}-\frac{u^{(1)}}{R}\right) \sin \varphi-\frac{\sin \varphi}{R}\right]+ \\
& +X_{3}\left[v_{2}^{(2)} \sin \varphi-\left(v_{3}^{(2)}-\frac{u^{(1)}}{R}\right) \cos \varphi+\frac{\cos \varphi}{R}-\frac{1}{R}\right]-\omega\left(\varphi^{(2)}+\frac{v_{2}^{(2)}}{R}\right)+\frac{1}{2} \frac{I_{p}}{A}\left(\varphi^{(1)}+\frac{v_{2}^{(1)}}{R}\right)^{2}
\end{aligned}
$$

This conforms to equation (6) [20]. The only difference is in the last part of expression of (5.4), which describes the Wagner's effect. In reference work of Pi, Bradford [20] it is not averaged for the cross-section, this, however is important only for substantially big rotations (Rosen [21]). In case of $1 / R=0$ the above formula simplifies to the one for straight rod [17, 18]. Taking into account Bernoulli hypothesis leads to simple relationships only in case of circular arches. If initial curvatures are dependent on $S$ arc-length coordinate, the formulas become very complicated [15]. It is due to fact, that in equation (4.3) we have to deal with derivatives of $\theta_{2}(S), \theta_{3}(S)$, and judging on equations (5.3) and (4.6bc) calculation of these derivatives in case, where $B, K_{2}$, and $K_{3}$ are functions of $S$, makes expression of equilibrium equations very complicated and for incremental approach, suitable for finite element formulation even more complex.

\section{CONCLUSIONS}

Contrary to appearances, variational formulation of the problem of spatially curved beams shown in Abaqus is very clear, what is confusing is merely the way it's dealing with finite rotations. On the other hand, formulations derived on basis of Bernoulli hypothesis usually lead to complicated formulas [15], so the promising idea seems to develop the one presented in [5] (Abaqus theory), 
choosing the right (second-order) approximations. Further the finite element method can be implemented - this is subject of consecutive works.

\section{REFERENCES}

1. S.P. Timoshenko, J.M. Gere, "Theory of elastic stability”, 2nd ed., McGraw-Hill, New York, 1961.

2. E. Reissner, "Some considerations on the problem of torsion and flexure of prismatical beams", Int. J. Solids Struct.,15:41-53, 1979.

3. E. Reissner, "On a simple variational analysis of small finite deformations of prismatical beams", J. Appl. Math. Phys. (ZAMP), 34:642-648, 1983.

4. J.H. Argyris, “An excursion into large rotation”, Comput. Meth. Appl. Mech. Engrg., 31: 85-155, 1982.

5. Abaqus 6.11, "Theory Manual”, Dassault Systemes, 2011.

6. E. Reissner, "On finite deformations of space-curved beams", J. Appl. Math. Phys. (ZAMP), 32:734-744, 1981.

7. A. Ibrahimbegovic', "On finite element implementation of geometrically nonlinear Reissner's beam theory: threedimensional curved beam elements“, Comput. Meth. Appl. Mech. Engrg., 122:11-26, 1995.

8. J.C. Simo, L. Vu-Quoc, “A geometrically exact rod model incorporating shear and torsion-warping”, Int. J. Solids Struct., 27(3):371-393, 1991.

9. F. Gruttmann, R. Sauer, W. Wagner, "Theory and numerics of three-dimensional beams with elastoplastic material behaviour", Int. J. Numer. Methods Engrg., 48:1675-1702, 2000.

10. J.M. Battini, C. Pacoste, "Co-rotational beam elements with warping effects in instability problems", Comput. Methods Appl. Mech. Engrg. 191: 1755-1789, 2002.

11. A.F. Saleeb, T.Y.P. Chang, A.S. Gendy, "Effective modelling of spatial buckling of beam assemblages accounting for warping constants and rotation-dependency of moments”, Int. J. Numer. Methods Engrg., 33:469-502, 1992.

12. M.Y. Kim, S.P. Chang, S.B. Kim, "Spatial stability analysis of thin-walled space frames", Int. J. Numer. Methods Engrg., 39:499-525, 1996.

13. A.F. Saleeb, A.S. Gendy, "Shear-flexible models for spatial bucking of thin-walled curved beams", Int. J. Numer. Methods Engrg., 31:729-57, 1991.

14. N. Hu, B. Hu, H. Fukunaga, H. Sekine, "Two kinds of $\mathrm{C}^{0}$ - type elements for buckling analysis of thin-walled curved beams", Comput. Meth. Appl. Mech. Engrg.,171:87-108, 1999.

15. P. F. Pai, "Geometrically exact beam theory without Euler angles”, Int. J. Solids Struct., 48: 3075-3090, 2011.

16. G.M. Van Erp, C.M. Menken, F.E. Veldpaus, "The nonlinear flexural torsional behaviour of straight slender elastic beam with arbitrary cross sections", Thin-Walled Struct., 6(5):385-404, 1988.

17. Y.L. Pi, N.S. Trahair, "Prebuckling deflections and lateral buckling - theory", J. Struct. Engng., ASCE, 118(11):2949-66, 1992.

18. F. Mohri, A. Eddinari, N. Damil, M. Potier-Ferry, "A beam finite element for non-linear analyses of thin-walled elements", Thin-Walled Struct., 46:981-90, 2008.

19. Y.L. Pi, N.S. Trahair, "Nonlinear elastic behaviour of I-beams curved in plan", J Struct. Engng., ASCE, 123(9):1201-1209, 1997.

20. Y.L. Pi, M.A. Bradford, "Elastic flexural-torsional buckling of continuously restrained arches", Int. J. Solids Struct., 39(8):2299-322, 2002 .

21. A. Rosen, "Theoretical and experimental investigation of nonlinear torsion and extension of initially twisted bars", J. Applied Mechanics, Trans. ASME, 21: 321-326, 1983.

\section{LIST OF FIGURES AND TABLES:}

Fig. 1. Initial and actual configuration of the rod.

Fig. 1. Konfiguracja pręta przed i po deformacji 


\section{O SKOŃCZONYCH ODKSZTALCENIACH BISYMETRYCZNYCH, ZAKRZYWIONYCH PRZESTRZENNIE BELEK O PRZEKROJU CIENKOSCIENNYM}

Slowa kluczowe: belki zakrzywione w przestrzeni, przekroje bisymetryczne, duże odkształcenia, aproksymacja skończonych obrotów, model Reissnera, hipoteza Bernoulliego

\section{STRESZCZENIE:}

W pracy przedstawiono geometrycznie nieliniową teorię wstępnie skręconych i zakrzywionych pryzmatycznych prętów cienkościennych o bisymetrycznym przekroju poprzecznym. Jest ona modyfikacją teorii przedstawionej w Abaqus Theory Manual [5]. Polega na zastąpieniu teorii [5] uwzględniającej, że w trakcie deformacji mogą wystąpić skończone obroty przekroju poprzecznego, inżynierską teorią ograniczoną do małych obrotów (ok. 0,3 rad). W konstrukcjach budowlanych, stan graniczny użytkowania wyklucza w praktyce pozostałe przypadki. To uproszczenie sprawia, że możemy sformułować inżynierską teorię, otrzymując wyniki zbliżone do otrzymanych w programie Abaqus [5]. Przedstawiono zmodyfikowane wzory na składowe odkształcenia Greena-Lagrange’a wstępnie skręconego i zakrzywionego w przestrzeni pręta cienkościennego przyjmując założenie, że 1) przekroje poprzeczne podlegające obrotom w przestrzeni w trakcie deformacji, nie muszą pozostawać prostopadłe do krzywej wyznaczonej przez środki ciężkości, 2) deformacja paczenia jest opisana przez niezależną od kąta skręcenia funkcję paczenia [5]. Do opisu obrotów przekroju poprzecznego wykorzystano aproksymację rzędu drugiego $(2.15,4.2)$. Prezentowane sformułowanie pozwala na wyprowadzenie praktycznie każdej z inżynierskich teorii prętów o przekroju cienkościennym przedstawionych w cytowanej literaturze problemu.

Rozpatrzono konfigurację pręta pokazaną na rysunku (rys.1), wprowadzając dwie bazy, pierwszą [ $\left.\mathbf{T}_{1}, \mathbf{T}_{2}, \mathbf{T}_{3}\right]$, w konfiguracji początkowej (niezdeformowanej) oraz drugą $\left[\mathbf{t}_{1}, \mathbf{t}_{2}, \mathbf{t}_{3}\right]$ dla przekroju po deformacji. System odniesienia w konfiguracji początkowej jest zbudowany w następujący sposób: wektor $\mathbf{T}_{1}$ jest zorientowany wzdłuz stycznej do krzywej odniesienia (będącej zbiorem punktów - środków ciężkości przekroju) nazywanej dalej osią pręta, wektory $\mathbf{T}_{2}$, $\mathbf{T}_{3}$ związane są zaś z głównymi kierunkami przekroju poprzecznego pręta. Układ zdeformowany, oznaczany małymi literami $\left[\mathbf{t}_{1}, \mathbf{t}_{2}, \mathbf{t}_{3}\right]$ jest budowany w analogiczny sposób, przy czym w ogólności wersor $\mathbf{t}_{1}$ nie musi pozostawać styczny do zdeformowanej krzywej odniesienia pręta. Zależność pomiędzy bazami $\left[\mathbf{T}_{i}\right]$ oraz [ $\mathbf{t}_{i}$ ] ma postać transformacji ortogonalnej (2.1). W tak określonych układach możemy zdefiniować położenie dowolnego punktu materialnego pręta przed deformacją (2.2) oraz po deformacji (2.3), przy czym przemieszczenie wywołane paczeniem przekroju określamy na kierunku wersora $\mathbf{t}_{1}$ jako iloczyn nieznanej amplitudy paczenia $p(S)$ i funkcji paczenia $\omega\left(X_{2}, X_{3}\right)$. W dalszej części pracy przedstawiono wzory na składowe uogólnionych odkształceń $\mathrm{w}$ bazie $\left[\mathbf{T}_{1}, \mathbf{T}_{2}, \mathbf{T}_{3}\right]$. Wzory na odkształcenie osiowe (2.10) wyprowadzono uśredniając części pochodzące od efektów Wagnera. W formułach określających naprężenia styczne wyodrębniono składniki pochodzące od sił poprzecznych i momentu skręcającego (2.11).

Transformację ortogonalną (2.1) wektorów bazowych $\left[\mathbf{T}_{i}\right]$ oraz $\left[\mathbf{t}_{i}\right]$ wykonujemy w sposób przybliżony za pomocą macierzy transformacji $\left[\boldsymbol{C}_{\mathrm{ij}}\right]$, będącej aproksymacją drugiego rzędu ścisłej macierzy obrotu $(2.15,4.2)$. Dzięki temu zamiast posługiwać się zaawansowaną algebrą kwaternionów opisującą skończone obroty w przestrzeni [5], mamy stosunkowo proste zależności dla uogólnionych odkształceń na osi pręta. W pracy przedstawiono dwie macierze transformacji (2.16 oraz 4.2). Macierz (4.2) otrzymano przez prostą aproksymację funkcji trygonometrycznych. Dzięki temu znacznie upraszczają się wzory na składowe tensora odkształcenia. Występujące w macierzy $(4.2)$ wyrazy $\varphi(S)$, $\theta_{2}(S), \theta_{3}(S)$ mogą być interpretowane jako obroty względem osi 1,2,3 (rys. 1) tylko w przypadku liniowej aproksymacji 
macierzy $\left[\boldsymbol{C}_{\mathrm{ij}}\right]$. Dla tak przedstawionego problemu sformułowano zasadę prac wirtualnych (3.12) definiując występujące w niej siły wewnętrzne wzorami (3.1-6), przy czym całkowity moment skręcający rozdzielono na moment skręcania St. Venanta oraz moment skręcania od paczenia zgodnie $\mathrm{z}$ formułami (3.7-3.10).

Pokazano, że teoria ta jest zgodna, po przyjęciu odpowiednich założeń upraszczających, z podejściem innych autorów z cytowanej literatury. W szczególności jest pewnym rozszerzeniem modelu Reissnera [6] uwzględniającym paczenie przekroju. W rozdziale 5 pokazano, że przyjęty model jest zgodny z pracą zaprezentowaną przez Pi, Bradford [20], z dokładnością do składników uwzględniających efekt Wagnera, które w pracy [20] nie są uśredniane dla przekroju co ma znaczenie jednak tylko w przypadku naprawdę dużych obrotów. Wnioskiem płynącym z pracy jest to, że przyjęcie hipotezy Bernoulliego prowadzi do prostych związków tylko dla przypadków stałej krzywizny pręta. W przypadku pręta o zmiennej krzywiźnie wzory stają się bardzo skomplikowane [15] ze względu na obecność pochodnych funkcji $\theta_{2}(S), \theta_{3}(S)$, których obliczanie, gdy $B, K_{2}$, and $K_{3}$ są funkcjami $S$ jest trudne, a sformułowanie przyrostowe MES bardzo skomplikowane. Przygotowany aparat obliczeniowy stanowi podstawę do rozwinięcia szczegółowego, bazującego na metodzie elementów skończonych, które pozwoli na jego dalsze badanie i praktyczną aplikację. Następnym krokiem badawczym jest sprawdzenie jaki wpływ na poprawność rozwiązania ma uśrednianie odkształceń normalnych od efektu Wagnera i jakie jest ograniczenie na kąt skręcenia dla tej metody. 